SUPPORTING INFORMATION FOR:

\title{
Solvent-Free Synthesis of High-Purity Sucrose Fatty Acid Mono-Esters and a Comparison of their Properties with those of their Commercial Counterparts
}

\author{
Meng-Fei Xie ${ }^{\dagger, t}$, Lorenzo V. White ${ }^{\ddagger}$, Martin G. Banwell, Yong Wang ${ }^{*}+,+$, \\ and Ping Lan*,t,
}

${ }^{\dagger}$ Guangdong Saskatchewan Oil Seed Joint Laboratory, Department of Food Science and Engineering, Jinan University, Guangzhou 510632, Guangdong, China

Institute for Advanced and Applied Chemical Synthesis, Jinan University, Guangzhou/Zhuhai, 510632/519070, Guangdong, China

*Corresponding authors (Y. W., Email: twyong@jnu.edu.cn, Tel.: +86-2085226326; P. L., E-mail: ping.lan@jnu.edu.cn, Tel: +86-20-85221367)

\section{CONTENTS}

Synthesis of standard samples 6- $O$-stearoylsucrose (1) and 6,6'-O-

stearoylsucrose (2)

${ }^{1} \mathrm{H}$ - and ${ }^{13} \mathrm{C}$-NMR Spectra of Compounds 1-2

The analytical plot (A) and calibration (B) curve of compounds 1, 2, and 
Synthesis of standard samples 6-O-stearoylsucrose (1) and 6,6'-O-stearoylsucrose (2)

6-O-stearoylsucrose, 1 . A solution of sucrose $(3.0 \mathrm{~g}, 8.7 \mathrm{mmol})$ in anhydrous THF/pyridine (50 mL, 1:1, v/v) was treated with lipozyme TLIM $(3.0 \mathrm{~g}, 100 \% \mathrm{w} / \mathrm{w}$ loading) and vinyl stearate (13.5 g, 5.0 eq., $43.5 \mathrm{mmol})$. The ensuing mixture was stirred magnetically at $50{ }^{\circ} \mathrm{C}$ for $48 \mathrm{~h}$ then cooled and the residual enzyme removed by filtration and the solid thus retained washed with dichloromethane $(2 \times 30 \mathrm{~mL})$ then methanol $(2 \times 30 \mathrm{~mL})$. The combined filtrates were dried $\left(\mathrm{Na}_{2} \mathrm{SO}_{4}\right)$, filtered then concentrated under reduced pressure to afford a yellow oil that was subjected to flash chromatography on silica and eluting with methanol/dichloromethane (1:9, v/v). Concentration of the relevant fractions $\left(R_{\mathrm{f}}=0.2\right)$ then afforded compound $\mathbf{1}(2.5 \mathrm{~g}, 47 \%)$ as an amorphous, white solid. ${ }^{1} \mathrm{H}$ NMR $\left(600 \mathrm{MHz}\right.$, methanol- $\left.d_{4}\right) \delta 5.38(\mathrm{~d}, J=3.8 \mathrm{~Hz}$, 1H, H-1), 4.40 (dd, $J=12.0,2.1 \mathrm{~Hz}, 1 \mathrm{H}, \mathrm{H}-6 \mathrm{a}), 4.17$ (dd, $J=12.0,5.4 \mathrm{~Hz}, 1 \mathrm{H}, \mathrm{H}-5)$, 4.09 (d, $J=8.4$ Hz, 1H, H-6b), $4.05-3.95$ (m, 2H, H-3', H-1'a), $3.84-3.75$ (m, 2H, H-6'a, H-6'b), 3.76 - 3.68 (m, 2H, H-1'b, H-2), 3.62 (d, J=12.3 Hz, 1H, 6'-OH), 3.58 (d, $J=12.3 \mathrm{~Hz}, 1 \mathrm{H}, 1-\mathrm{OH}), 3.42(\mathrm{dd}, J=9.8,3.8 \mathrm{~Hz}, 1 \mathrm{H}, \mathrm{H}-3), 3.31-3.26$ (m, 1H, H4), 2.37 (t, $\left.J=7.5 \mathrm{~Hz}, 2 \mathrm{H},-\underline{\mathrm{C}}_{2}-\mathrm{CO}-\right), 1.62$ (p, $\left.J=7.3 \mathrm{~Hz}, 2 \mathrm{H},-\underline{\mathrm{H}}_{2}-\mathrm{CH}_{2}-\mathrm{CO}-\right), 1.37$ $-1.29\left(\mathrm{~m}, 9 \mathrm{H},-\mathrm{CH}_{2}-\right), 1.29\left(\mathrm{~s}, 20 \mathrm{H},-\mathrm{CH}_{2}-\right), 0.90\left(\mathrm{t}, J=7.0 \mathrm{~Hz}, 3 \mathrm{H},-\mathrm{CH}_{3}\right) .{ }^{13} \mathrm{C} \mathrm{NMR}$ (151 MHz, methanol- $\left.d_{4}\right) \delta 175.39(\mathrm{C}=\mathrm{O}), 105.10\left(\mathrm{C} 2^{\prime}\right), 93.27(\mathrm{C} 1), 83.77\left(\mathrm{C}^{\prime}\right), 79.11$ (C3'), 75.80 (C4'), 74.36 (C3), 73.03 (C2), 71.84 (C5), 71.52 (C4), 64.55 (C6), 63.99 (C1'), $63.81\left(\mathrm{C6}^{\prime}\right), 34.80\left(-\mathrm{CH}_{2}-\mathrm{CO}-\right), 32.94,30.65(\times 6), 30.62(\times 2), 30.51,30.33(\times 2)$, 30.10, 25.88, $23.59\left(-\mathrm{CH}_{2}-\mathrm{CH}_{3}\right), 14.30\left(-\mathrm{CH}_{3}\right) . \mathrm{MS}(\mathrm{ESI},+\mathrm{ve}): \mathrm{m} / z 626\left[\mathrm{M}+\mathrm{NH}_{4}{ }^{+}\right]^{+}$

6,6'- $\boldsymbol{O}$-stearoylsucrose, 2. A solution of mono-ester $1(2.0 \mathrm{~g}, 3.3 \mathrm{mmol})$ in 
anhydrous acetone $(80 \mathrm{~mL})$ was treated with Novozym $435(2.0 \mathrm{~g}, 100 \% \mathrm{w} / \mathrm{w}$ loading $)$ and vinyl stearate $(5.1 \mathrm{~g}, 5.0$ eq., $16.5 \mathrm{mmol})$. The ensuing mixture was stirred magnetically at $45{ }^{\circ} \mathrm{C}$ for $24 \mathrm{~h}$ then cooled and the residual enzyme removed by filtration and the solid thus retained washed with dichloromethane $(2 \times 30 \mathrm{~mL})$ then methanol $(2 \times 30 \mathrm{~mL})$. The combined filtrates were dried $\left(\mathrm{Na}_{2} \mathrm{SO}_{4}\right)$, filtered then concentrated under reduced pressure to afford a yellow oil that was subjected to flash chromatography on silica and eluting with methanol/dichloromethane $(1: 9, \mathrm{v} / \mathrm{v})$. Concentration of the relevant fractions $\left(R_{\mathrm{f}}=0.4\right)$ then afforded compound $2(1.8 \mathrm{~g}, 63 \%)$ as an amorphous, white solid. ${ }^{1} \mathrm{H}$ NMR $\left(600 \mathrm{MHz}, \mathrm{DMSO}-d_{6}\right) \delta 5.37(\mathrm{~d}, J=5.8 \mathrm{~Hz}$, $1 \mathrm{H}), 5.10(\mathrm{dd}, J=6.9,4.8 \mathrm{~Hz}, 2 \mathrm{H}), 5.04(\mathrm{~d}, J=5.6 \mathrm{~Hz}, 1 \mathrm{H}), 4.88(\mathrm{~d}, J=4.9 \mathrm{~Hz}, 1 \mathrm{H})$, $4.83(\mathrm{t}, J=6.4 \mathrm{~Hz}, 1 \mathrm{H}), 4.73(\mathrm{~d}, J=7.8 \mathrm{~Hz}, 1 \mathrm{H}), 4.33-4.26(\mathrm{~m}, 2 \mathrm{H}), 4.15(\mathrm{dd}, J=$ 11.7, $8.4 \mathrm{~Hz}, 1 \mathrm{H}), 3.94-3.86(\mathrm{~m}, 3 \mathrm{H}), 3.79(\mathrm{td}, J=8.3,5.8 \mathrm{~Hz}, 1 \mathrm{H}), 3.73(\mathrm{td}, J=8.3$, $2.7 \mathrm{~Hz}, 1 \mathrm{H}), 3.48(\mathrm{td}, J=9.2,4.5 \mathrm{~Hz}, 1 \mathrm{H}), 3.41-3.34(\mathrm{~m}, 2 \mathrm{H}), 3.20(\mathrm{ddd}, J=9.7,6.0$, $3.7 \mathrm{~Hz}, 1 \mathrm{H}), 3.00(\mathrm{td}, J=9.2,5.3 \mathrm{~Hz}, 1 \mathrm{H}), 2.29(\mathrm{dt}, J=18.4,7.5 \mathrm{~Hz}, 4 \mathrm{H}), 1.50(\mathrm{q}, J=$ $6.8 \mathrm{~Hz}, 4 \mathrm{H}), 1.29-1.21(\mathrm{~m}, 56 \mathrm{H}), 0.85(\mathrm{t}, J=6.9 \mathrm{~Hz}, 6 \mathrm{H}) .{ }^{13} \mathrm{C}$ NMR $(151 \mathrm{MHz}$, DMSO- $\left.d_{6}\right) \delta 172.59,103.90,91.27,78.98,74.63,72.39,71.27,70.27,69.94,65.54$ $64.00,61.73,33.14,33.06,31.16,28.95,28.93,28.87,28.85,28.80,28.66,28.62,28.58$, 28.38, 28.34, 24.27, 21.96, 13.79. MS (ESI, +ve): $m / z 892\left[\mathrm{M}+\mathrm{NH}_{4}{ }^{+}\right]^{+}$ 
$600 \mathrm{MHz}{ }^{1} \mathrm{H}$ NMR Spectrum of Compound 1 (Recorded in Methanol- $d_{4}$ )

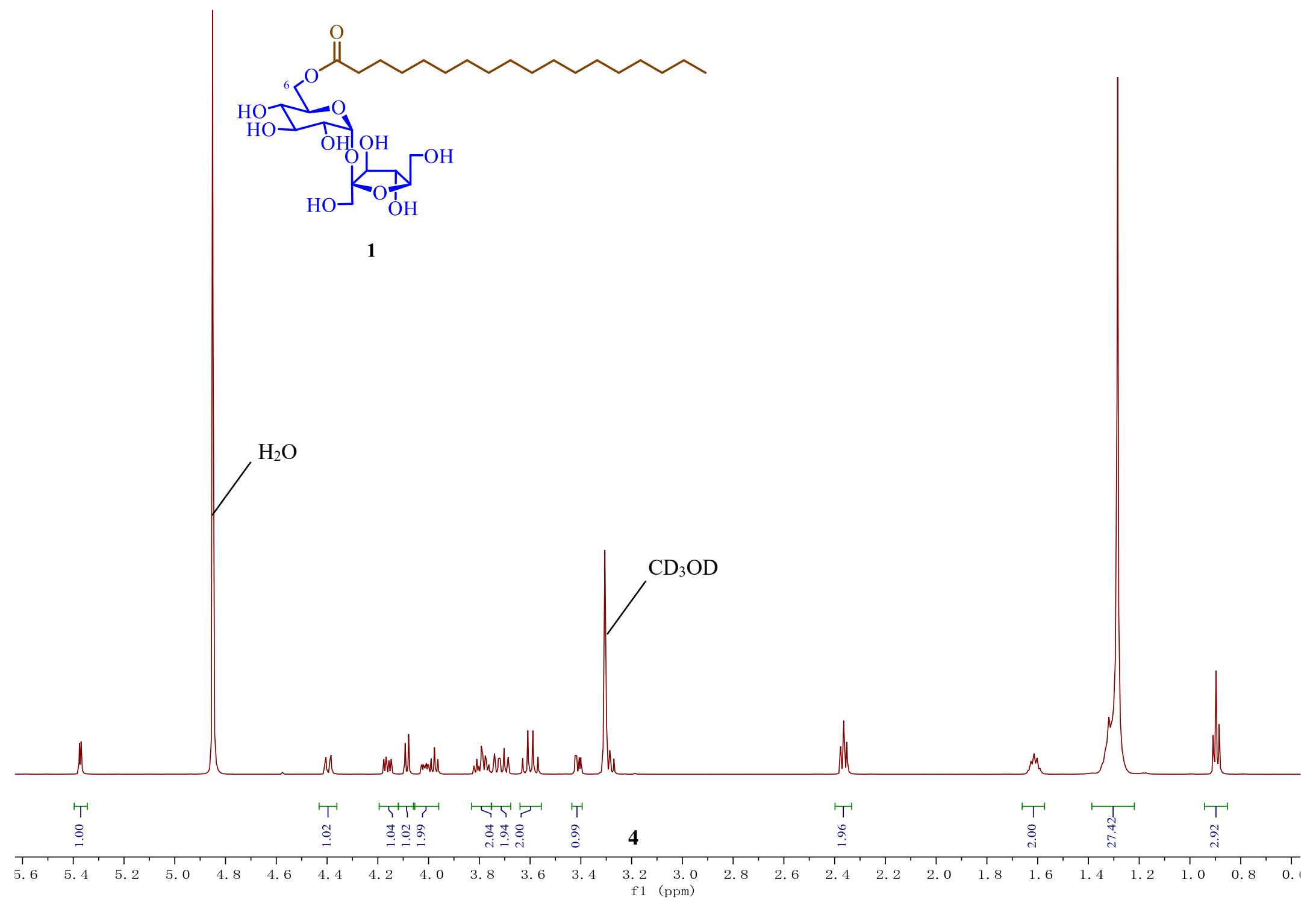




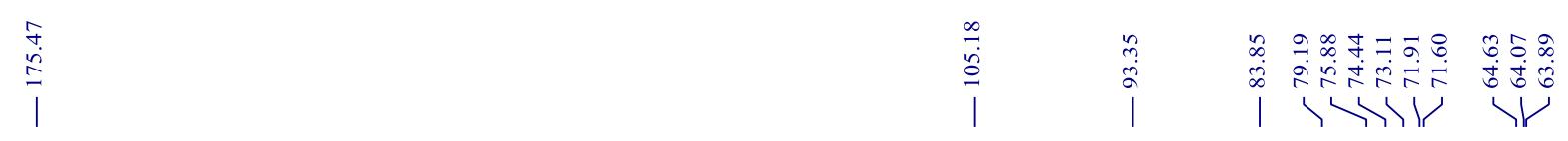

$150 \mathrm{MHz}{ }^{13} \mathrm{C}$ NMR Spectrum of Compound 1 (Recorded in Methanol- $\left.d_{4}\right)$

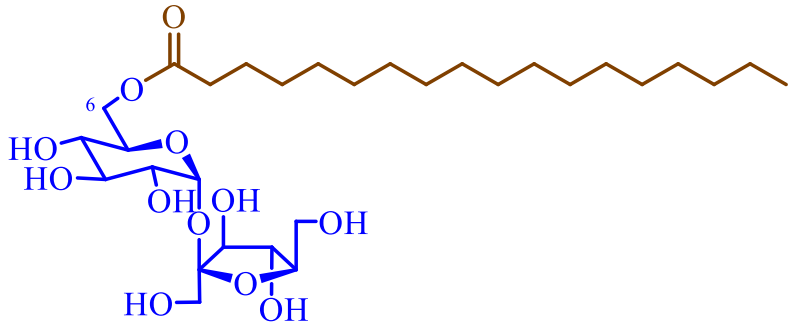

1

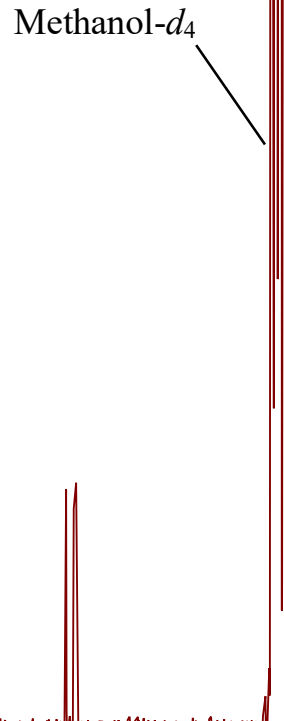

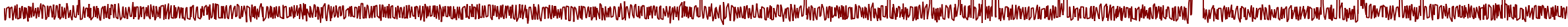

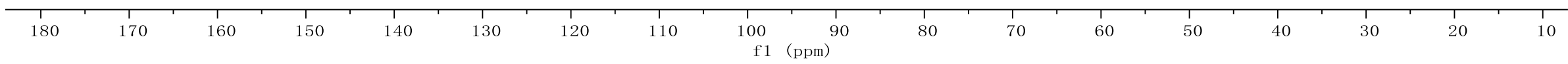


$600 \mathrm{MHz}^{1} \mathrm{H}$ NMR Spectrum of Compound 2 (Recorded in DMSO- $d_{6}$ )

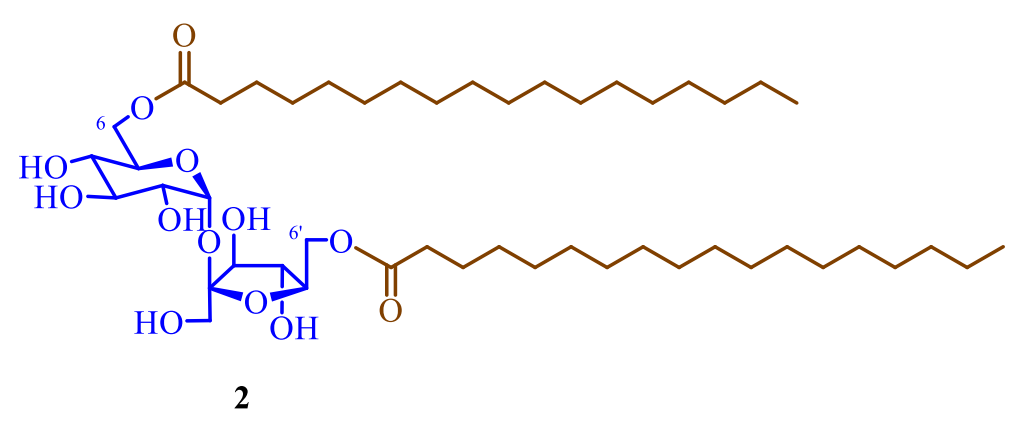

2

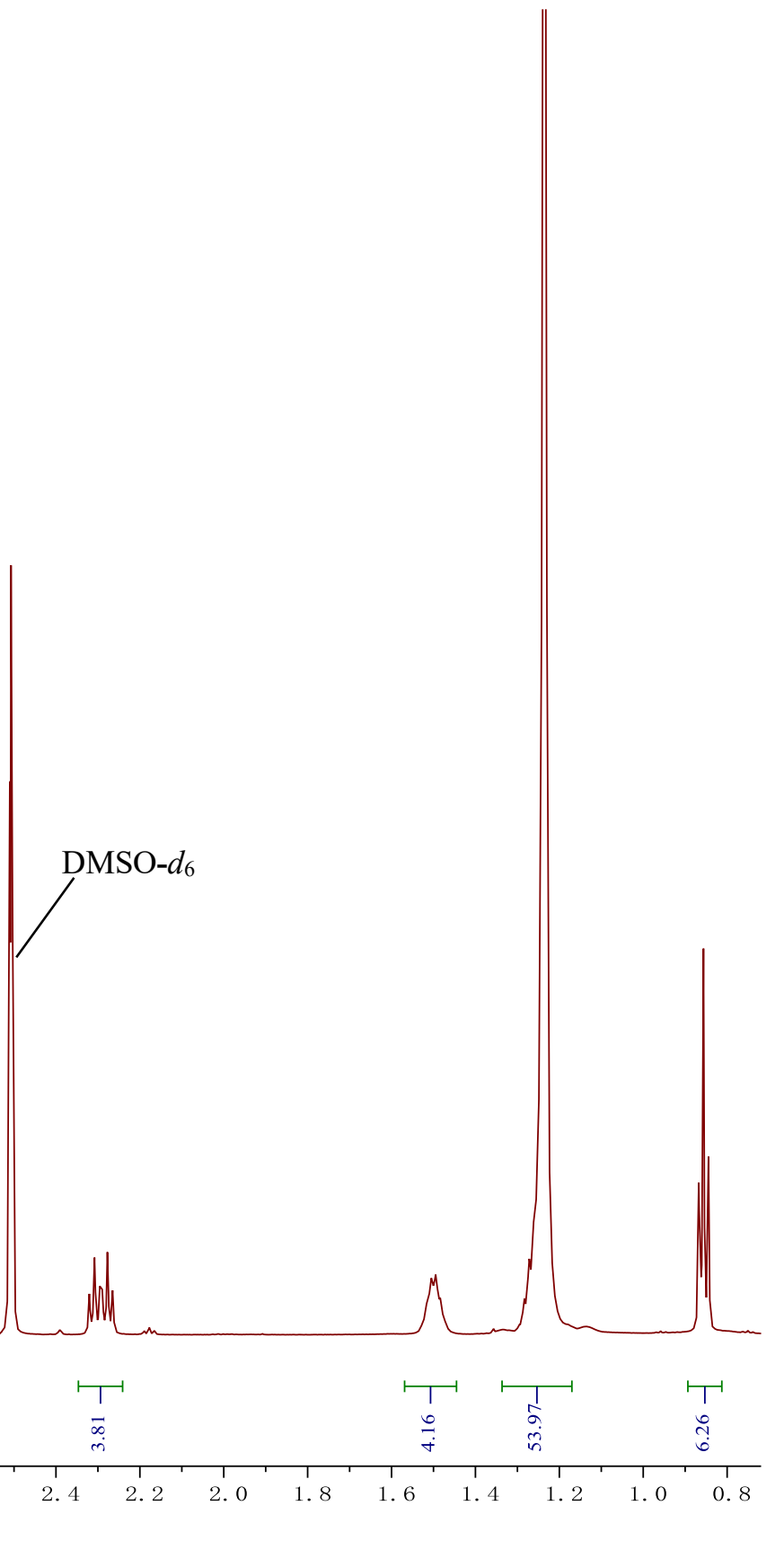




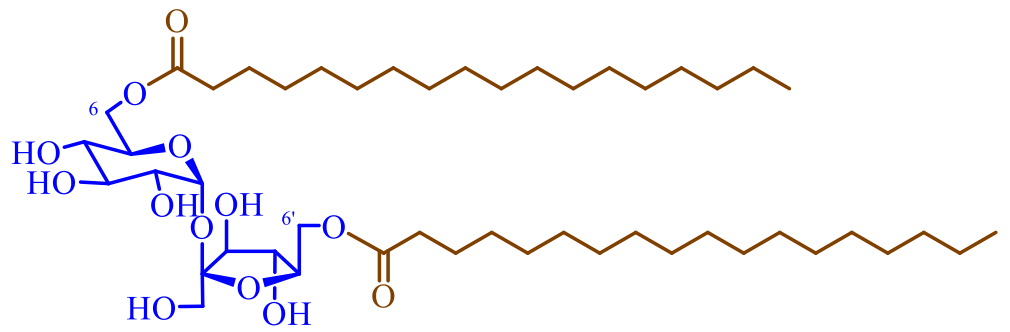

2

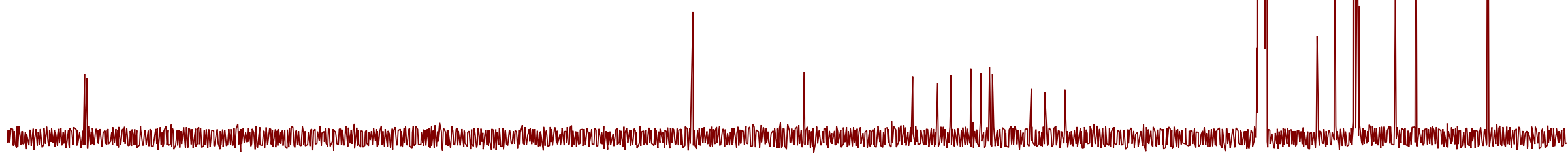
7 


\section{SIR of 1 channel,ES+}

626.38

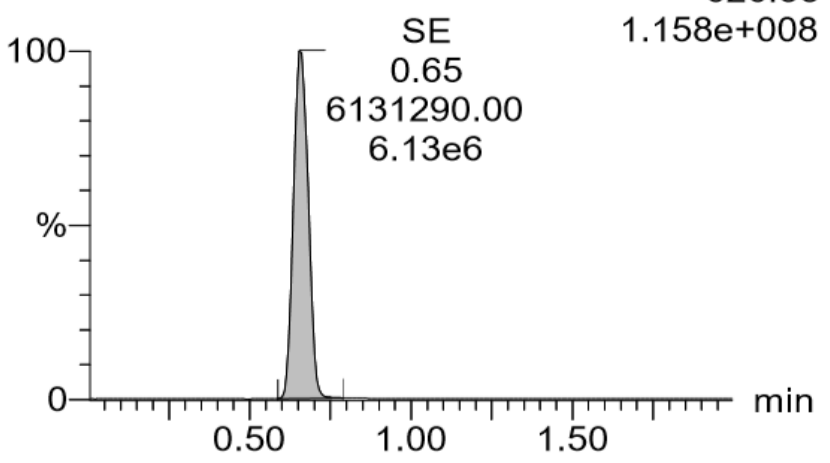

A

Compound name: SE

Correlation coefficient: $r=0.996994, r^{\wedge} 2=0.993997$

Calibration curve: $3.6768 \mathrm{e}+007{ }^{*} \mathrm{x}+368431$

Response type: External Std, Area

B

Curve type: Linear, Origin: Exclude, Weighting: Null, Axis trans: None
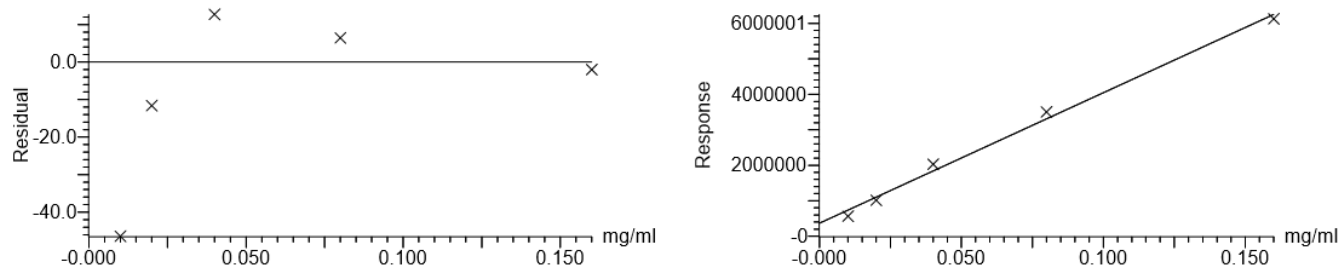

The analytical plot (A) and calibration curve of sucrose stearic acid mono-ester (B) $(0.01-0.16 \mathrm{mg} / \mathrm{mL})$ 
12-3-DISSE-016C-Tg SIR of 1 channel,ES+

892.6725

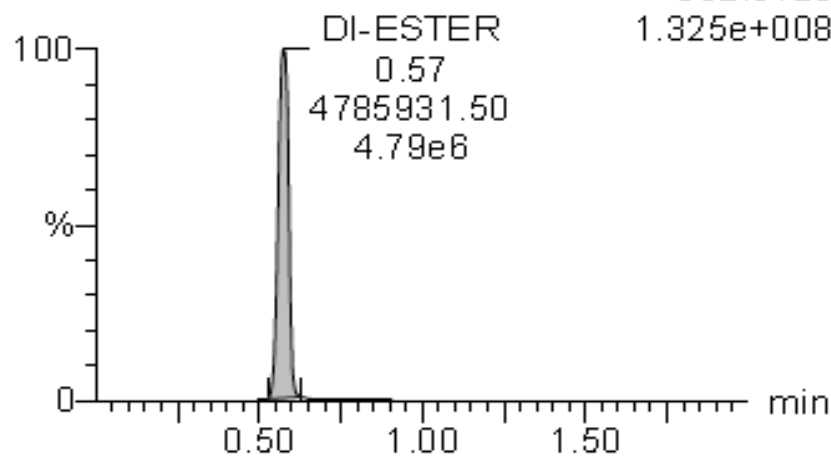

A

B

Compound name: DI-ESTER

Calibration curve: $2.81721 \mathrm{e}+007^{*} \mathrm{x}+441305$

Response type: External Std, Area

Curve type: Lin : Origin: Exclude, Weighting: 1/x, Axis trans: None
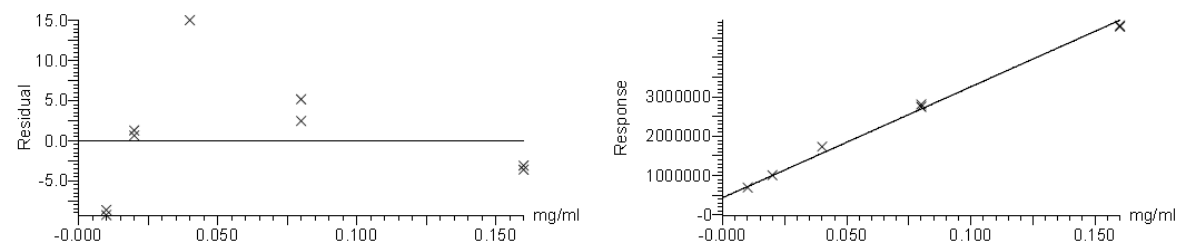

The analytical plot (A) and calibration curve of sucrose stearic acid di-ester (B) (0.01$0.16 \mathrm{mg} / \mathrm{mL}$ ) 


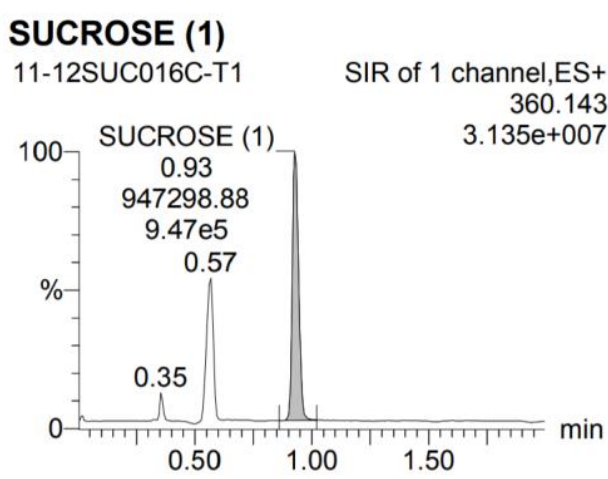

A

Method: D: Xie Mengfei.PROIMeth Calibration: 14 Aug 2020 11:46:39

Compound name: SUCROSE (1)

Correlation coefficient: $r=0.998394, r^{\wedge} 2=0.996791$

Calibration curve: $5.55409 \mathrm{e}+006^{*} \mathrm{x}+-32769.3$

Response type: External Std, Area

B

Curve type: Linear, Origin: Exclude, Weighting: 1/x, Axis trans: None
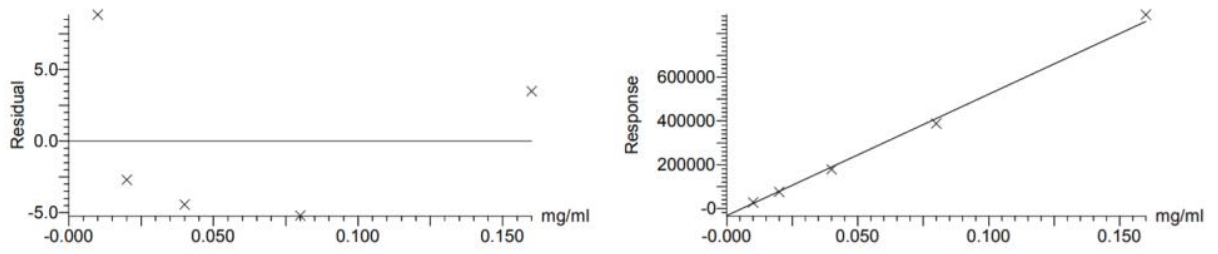

The analytical plot (A) and calibration curve of sucrose $(0.01-0.16 \mathrm{mg} / \mathrm{mL})(\mathbf{B})$ 


\section{ME-ST}

ME004-T1

SIR of 1 channel,ES+

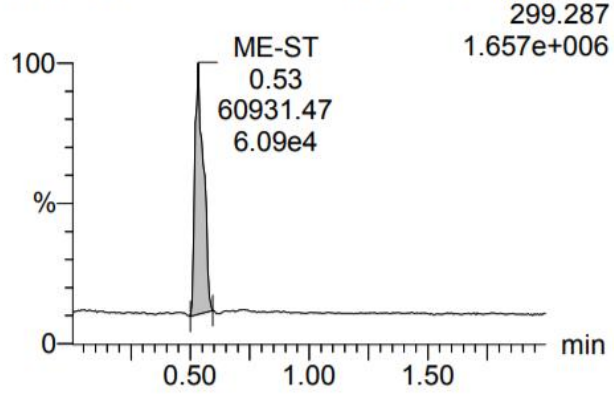

A

Compound name: ME-ST

Correlation coefficient: $r=0.995306, r^{\wedge} 2=0.990633$

Calibration curve: $581635^{*} \mathrm{x}+24366.2$
Response type: External Std, Area

Curve type: Linear, Origin: Exclude, Weighting: 1/x, Axis trans: None

B
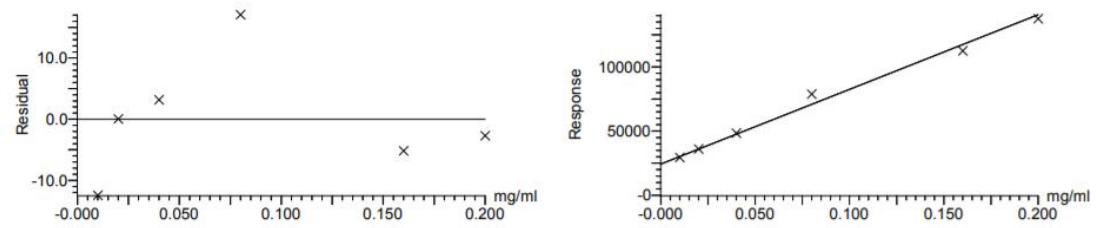

The analytical plot (A) and calibration curve of sucrose stearate $(\mathbf{B})(0.01-0.16$ $\mathrm{mg} / \mathrm{mL})$ 